\title{
Food insecurity and emotional health in the USA: a systematic narrative review of longitudinal research
}

\author{
Meg Bruening ${ }^{1, *}$, Lauren M Dinour ${ }^{2}$ and Jose B Rosales Chavez ${ }^{3}$ \\ ${ }^{1}$ School of Nutrition and Health Promotion, Arizona State University, 500 N. 3rd Street, Phoenix, AZ 85004, USA: \\ ${ }^{2}$ Department of Nutrition and Food Studies, Montclair State University, Montclair, NJ, USA: ${ }^{3}$ School of Human \\ Evolution and Social Change, Arizona State University, Phoenix, AZ, USA
}

Submitted 3 February 2017: Final revision received 13 June 2017: Accepted 23 June 2017: First published online 14 September 2017

\begin{abstract}
Objective: To examine the causal directionality in the relationship between food insecurity and emotional well-being among US-based populations.

Design: Systematic literature review from January 2006 to July 2016 using MEDLINE (PubMed), PsychInfo, Web of Science and CINHAL. Inclusion criteria were: written in English; examined a longitudinal association between food insecurity and emotional well-being.

Setting: The USA.

Subjects: Children and adults.

Results: Twelve out of 4161 peer-reviewed articles met inclusion criteria. Three articles examined the effect of emotional well-being on food insecurity, five studies examined the effect of food insecurity on emotional well-being, and four studies examined a bidirectional relationship. Most studies (83\%) reported a positive relationship between negative emotional well-being and food insecurity over time. Conclusions: Findings suggest a bidirectional association whereby food insecurity increases the risk of poor emotional health, and poor emotional health increases the risk of food insecurity. Better-constructed studies are needed to follow cohorts at risk for both food insecurity and poor emotional health to further understand the mediators and moderators of the relationships. Intervention studies designed to mitigate or reverse risks are also needed to determine best evidence for practice and policy.
\end{abstract}

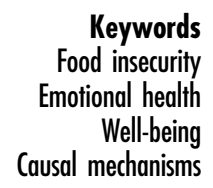

Keywords

Food insecurity

Well-being

Causal mechanisms
In the USA in 2015, the prevalence of food insecurity, or inconsistent access to healthy foods, was $12 \cdot 7 \%$, affecting almost 16 million households ${ }^{(1)}$. Women, children and people of colour are disproportionately impacted by food insecurity $^{(2,3)}$. Food insecurity has been related to lower productivity $^{(4)}$ and academic outcomes ${ }^{(5)}$, poorer nutritional health ${ }^{(6,7)}$ and higher rates of chronic disease such as diabetes ${ }^{(8,9)}$. Those who struggle with food insecurity often report struggling with mental health ${ }^{(10-14)}$.

Concurrent food insecurity and emotional health and well-being has been examined significantly in crosssectional research. Overwhelmingly, the literature has shown that food insecurity is related to higher levels of depression, stress and anxiety. For example, HromiFiedler et al. reported over 2.5 higher odds of depression among pregnant food-insecure women compared with pregnant food-secure women ${ }^{(10)}$, a finding which is supported by others ${ }^{(11,12)}$. Data from the National Health and Nutrition Examination Survey suggest that food insecurity, but not poverty, is associated with higher rates of depression and even suicide ideation and attempts among adolescents $^{(13)}$. In a review of the twenty-seven qualitative and quantitative studies from developing nations, Weaver and Hadley reported that food insecurity is related to anxiety, shame, stress, resignation and depression ${ }^{(14)}$. However, a clear understanding on how food insecurity differentially impacts emotional well-being over time in the USA is needed. Food insecurity and emotional wellbeing may manifest differently in the USA, as access to public programmes and perceptions of health may vary from developing and other high-income nations.

Given that most of the research examining food insecurity and emotional health and well-being is crosssectional, we need more insight into the causal mechanisms involved in the associations. By understanding the temporality, interventions can be better designed to assist those 
struggling. As such, the purpose of the present study was to conduct a systematic narrative review of the longitudinal literature assessing the relationship between food insecurity and emotional health and well-being. We limited our search to the USA to inform intervention work for those populations, differentiating the present review from the recent review conducted in developing nations ${ }^{(14)}$.

\section{Methods}

A systematic review of recent literature (January 2006-July 2016) was conducted to find articles that addressed food insecurity and emotional health over time. A combination of food insecurity and emotional health key terms (Table 1) was used to create a comprehensive list of articles from MEDLINE (PubMed), PsychInfo, Web of Science and CINHAL databases. English-language studies were screened for food insecurity and emotional health. Studies were included for review if they were observational or intervention studies, and if they had individual or household food insecurity measures, and any measure, positive or negative, of emotional health. Studies were limited to longitudinal designs conducted in the USA. Cross-sectional and qualitative studies were excluded.

Articles were reviewed in the following order: title, abstract, methods and full manuscript. M.B. and L.M.D. reviewed all titles and abstracts and identified articles that met the inclusion criteria. J.B.R.C. reviewed all identified articles and settled disagreements between M.B. and L.M.D. Data extraction for each article meeting the inclusion criteria was completed by the three researchers independently and compared for full accuracy. Extracted data included: authors' names, year of publication, data source, year of data collection, study time points used for the analysis, country of study, sample demographics, populations included in the study as stage of the life cycle, relevant food insecurity and emotional health measures, directionality of the relationships, outcomes related to food insecurity and emotional health, and limitations as identified by the author. The reference lists of articles meeting inclusion criteria were screened for additional studies. We used the PRISMA (Preferred Reporting Items

Table 1 Food insecurity and emotional well-being key terms

\begin{tabular}{ll}
\hline Food insecurity key terms & Emotional health key terms \\
\hline Food insecurity & Depression \\
Food hardship & Stress \\
Food insufficiency & Anxiety \\
Inconsistent food sources & Cortisol \\
Hunger & Mood \\
& Anger \\
& Happiness \\
& Satisfaction \\
& Contentment \\
& Frustration \\
& Angry \\
& Emotion \\
\hline
\end{tabular}

for Systematic Reviews and Meta-Analyses) checklist for the systematic review.

\section{Results}

In total, after duplicates were removed, 4161 articles were screened at the title and abstract levels (see Fig. 1). Of these, only seventeen articles were screened at the fulltext level. The present literature review includes the findings from twelve longitudinal studies assessing measures of food insecurity and emotional well-being (Table 2). While all but two of the twelve studies examined the longitudinal relationships between food insecurity and emotional well-being in households with children, only one study examined child emotional well-being outcomes $^{(15)}$. Descriptions of measurements and data sources are included in the online supplementary material, Supplemental Tables 1 and 2, respectively.

\section{Effect of emotional well-being on food insecurity}

Three articles looked solely at the relationship between depression at baseline and food insecurity at followup $^{(16-18)}$. All three measured food security status via the eighteen-item US Department of Agriculture Household Food Security Survey Module (USDA HFSSM); although the measure for depression differed between the Center for Epidemiologic Studies Depression (CES-D) twentyitem scale ${ }^{(16)}$, the CES-D twelve-item scale ${ }^{(17)}$ and the Composite International Diagnostic Interview Short Form (CIDI-SF $^{(18)}$. Among the three articles, samples of parents with young children were drawn from three different data sources $^{(16-18)}$. The analyses by Hernandez et al. and Garg et al. focused exclusively on maternal depression ${ }^{(17,18)}$ whereas Hanson and Olson examined depression among parents ${ }^{(16)}$. The mean sample size among the three studies was 1611 (range: 225-2917).

Despite their different measures and samples, these three articles showed the same general outcome pattern: depression at baseline is associated with food insecurity at follow-up. To illustrate, Hernandez et al. found that households in which mothers experienced depression were twice as likely to experience food insecurity $(\mathrm{OR}=2 \cdot 03 ; P<0.001)$ compared with households in which mothers did not experience depression ${ }^{(18)}$. This relationship also remained after controlling for intimate partner violence (adjusted $\mathrm{OR}=1.97 ; P<0.001)^{(18)}$. Similarly, in the article by Garg et al. maternal depression at baseline predicted household food insecurity at follow-up (adjusted $\mathrm{OR}=1 \cdot 50,95 \%$ CI $1 \cdot 06,2 \cdot 12$ ) after controlling for sociodemographics and maternal self-reported health status ${ }^{(17)}$.

In the article by Hanson and Olson, compared with respondents having no years at risk for depression, respondents with 2 years of depression risk were significantly more likely to have persistent food insecurity (food insecurity for three straight years) than to have no 


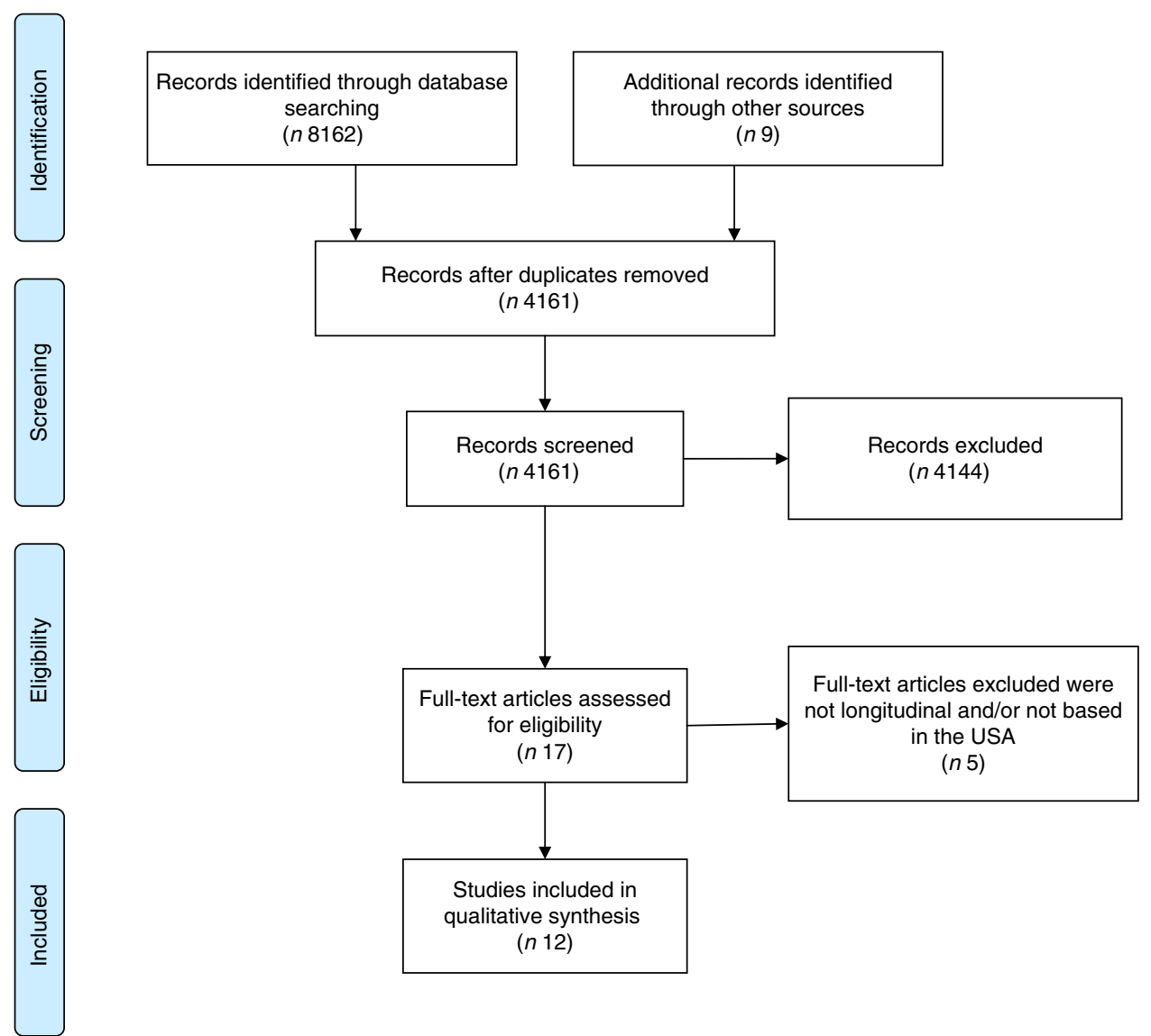

Fig. 1 Flow diagram of peer-reviewed literature examining the longitudinal relationship between food insecurity and emotional well-being

food insecurity $(\mathrm{OR}=4 \cdot 28 ; P<0 \cdot 01)$ or discontinuous food insecurity (food insecurity for 1 or 2 years, $\mathrm{OR}=3.65$; $P<0 \cdot 05)^{(16)}$.

\section{Effect of food insecurity on emotional well-being}

Five studies hypothesized that food insecurity was a factor driving emotional well-being ${ }^{(15,19-22)}$. The populations examined in these analyses covered virtually all periods of the life course, from pregnancy/postpartum, toddlers and adolescents to the elderly ${ }^{(15,19-22)}$. The mean sample size for these analyses was 4755 (range: 416-9481). The most commonly used tool to measure food insecurity was the USDA HFSSM ${ }^{(19,20,22)}$. Other food insecurity measures included the Core Food Security Module ${ }^{(21)}$ and questionnaires generated by investigators ${ }^{(15)}$.

For emotional well-being, most studies measured the mothers' depression levels ${ }^{(15,19,20)}$. Several studies examined anxiety ${ }^{(15)}$ and stress ${ }^{(21,22)}$. Two studies also included measures for children's emotional well-being by measuring the children's internalizing and externalizing behaviours $^{(15,22)}$. Internalizing behaviours included withdrawn or sad behaviours, and externalizing behaviours included aggressive behaviours such as disobedience, anger and defiance ${ }^{(15,22)}$. Emotional health was measured using a wide variety of tools: CES-D eight-item scale ${ }^{(20)}$; CES-D twenty-item scale ${ }^{(19)}$; Child Behavior Checklist for anxiety/ depression, withdrawn and aggressive subscales for mothers and children ${ }^{(15)}$; ten-item Perceived Stress Scale ${ }^{(21)}$; Emotional Wellbeing: Toddler Attachment Sort- $45^{(19)}$; and the thirty-two-item Behavior Problem Index for internalizing and externalizing behaviours ${ }^{(22)}$.

Each study drew samples of adults and children from different sources: Health and Retirement Study (HRS) ${ }^{(20)}$; Asset and Health Dynamics Among the Oldest Old $(\mathrm{AHEAD})^{(20)}$; Fragile Families and Child Well-being Study $(\text { FFCWB })^{(15)}$; Pregnancy, Infection and Nutrition (PIN) study $^{(21)}$; Early Childhood Longitudinal Study-Birth Cohort (ECLS-B) ${ }^{(19)}$; and Child Development Supplement (CDS) to the Panel Study of Income Dynamics (PSID) ${ }^{(22)}$.

The overall results supporting the hypothesis that food insecurity drives depression levels were mixed. Over half ( $60 \%$ ) of the studies found that food insecurity at baseline is associated with emotional health at follow-up ${ }^{(19-21)}$. This can be illustrated by Laraia et al., who found that women who were exposed to any level of food insecurity during pregnancy and postpartum had high levels of perceived stress at 3 and 12 months postpartum $(\beta=3 \cdot 36,95 \% \mathrm{CI}$ $0.79,5.92 ; \beta=3.67,95 \%$ CI $0.94,6.41$, respectively) ${ }^{(21)}$. Likewise, Kim and Frongillo found that among one group of younger elders (mean age $=60.8$ years), those who 
Table 2 Data extraction of longitudinal US studies that examine the relationship between food insecurity (FI) and emotional well-being

\begin{tabular}{|c|c|c|c|c|c|c|c|c|}
\hline \multirow[b]{2}{*}{ Study } & \multirow[b]{2}{*}{ Study time points } & \multirow[b]{2}{*}{ Data set } & \multirow[b]{2}{*}{ Sample and demographics } & \multicolumn{2}{|c|}{ Measures } & \multicolumn{2}{|c|}{ Prevalence at baseline } & \multirow[b]{2}{*}{ Outcomes } \\
\hline & & & & $\mathrm{FI}$ & Emotional well-being & $\mathrm{FI}$ & Emotional well-being & \\
\hline \multicolumn{9}{|c|}{ Effect of emotional well-being on food insecurity } \\
\hline $\begin{array}{l}\text { Hanson and Olson } \\
(2012)^{(16)}\end{array}$ & $\begin{array}{l}\text { T1: } 1999 \\
\text { T2: } 2003\end{array}$ & $\begin{array}{l}\text { Rural Low-income Families: } \\
\text { Monitoring their Well-being and } \\
\text { Functioning in the Context of } \\
\text { Welfare Reform }\end{array}$ & $\begin{array}{l}\text { Parents, } n 225 \\
\% \text { Female }=\text { NA } \\
\text { Mean age }=30.6 \text { years } \\
\text { Race/ethnicity }=33.8 \% \text { non-white } \\
\text { Mean incomelhousehold } \\
\text { poverty }=\text { NA }\end{array}$ & $\begin{array}{l}\text { Eighteen-item USDA } \\
\text { HFSSM }\end{array}$ & $\begin{array}{l}\text { Twenty-item CES-D } \\
\text { scale: never, one } \\
\text { year or two years at } \\
\text { risk for depression }\end{array}$ & $51 \cdot 1 \%$ & NA & $\begin{array}{l}\text { Length of depression was significantly associated with } \\
\text { persistent } \mathrm{FI} \text {, but not discontinuous } \mathrm{FI} \\
\text { - Discontinuous } \mathrm{FI} \text { : } \\
\text { o } 1 \text { year at risk for depression (FI OR=1.03; } P>0.05) \\
\text { } 2 \text { years at risk for depression }(\mathrm{FIOR}=1.17 ; P>0.05) \\
\text { - Persistent } \mathrm{Fl} \text { : } \\
\text { o } 1 \text { year at risk for depression }(\mathrm{FIOR}=1.57 ; P>0.05) \\
\text { - } 2 \text { years at risk for depression }(\mathrm{FIOR}=4.28 ; P<0.05)\end{array}$ \\
\hline $\begin{array}{l}\text { Hernandez et al. } \\
(2013)^{(18)}\end{array}$ & $\begin{array}{l}\text { T1: } 2001-2003 \\
(3 \text { years }) \\
\text { T2: 2003-2005 } \\
\text { (5 years) }\end{array}$ & $\begin{array}{l}\text { Fragile Families and Child } \\
\text { Well-Being Study and } \\
\text { In-Home Longitudinal Study } \\
\text { of Preschool Age Children }\end{array}$ & $\begin{array}{l}n 1690 \\
\% \text { Female }=100 \\
\text { Mean age }=28.4 \text { (sD } 6.0) \text { years } \\
\text { Race/etthicity }=26 \% \text { white, } 44 \% \\
\text { black, } 26 \% \text { Hispanic, } 3 \% \text { other } \\
\text { race } 26 \text {. } \\
\text { Mean income/household } \\
\text { poverty }=36 \% \text { at }<100 \% \mathrm{FPL}, \\
25 \% \text { between } 100 \text { and } 199 \% \\
\text { FPL, 39\% at }>200 \% \mathrm{FPL}\end{array}$ & $\begin{array}{l}\text { Eighteen-item USDA } \\
\text { HFSSM }\end{array}$ & $\begin{array}{l}\text { Composite } \\
\text { International } \\
\text { Diagnostic } \\
\text { Interview Short } \\
\text { Form for } \\
\text { Depression }\end{array}$ & $15 \%$ & $\%$ depressed & $\begin{array}{l}\text { Mothers' depression was significantly associated with } \\
\text { subsequent } \mathrm{FI}(\mathrm{aOR}=2.03,95 \% \mathrm{Cl} 1.45,2.84 ; \\
P<0.001) \\
\circ \text { After adding intimate partner violence into the model, } \\
\text { the relationship remained significant (aOR }=1.97 \text {, } \\
95 \% \mathrm{Cl} 1.41,2.76 ; P<0.001) \\
\text { - Depression fully mediated the relationship between } \\
\text { intimate partner violence and } \mathrm{FI} \text { (Sobel test }=2.89 \text {; } \\
P<0.01 \text { ) }\end{array}$ \\
\hline Garg et al. (2015) & $\begin{array}{l}\text { T1: 2001-2002 } \\
\text { (9 months) } \\
\text { T2: } 2003 \\
\text { (24 months) }\end{array}$ & $\begin{array}{l}\text { Early Childhood Longitudinal } \\
\text { Study, Birth Cohort }\end{array}$ & $\begin{array}{l}\text { n } 2917 \\
\% \text { Female }=100 \\
\text { Mean age }=25.5 \text { (sD } 5.8) \text { years } \\
\text { Race/ethnicity }=37.5 \% \mathrm{NH} \text { white, } \\
22.5 \% \text { NH black, } 34.8 \% \\
\text { Hispanic, } 2.1 \% \text { Asian/Pacific } \\
\text { Islander NH, } 3.1 \% \text { other } \\
\text { Mean income/household } \\
\text { poverty }=52.4 \% \text { at }<100 \% \\
\text { poverty threshold, } 24.2 \% \\
\text { between } 100 \text { and }<130 \% \text { poverty } \\
\text { threshold, } 23.3 \% \text { between } 130 \\
\text { and }<185 \% \text { poverty threshold }\end{array}$ & $\begin{array}{l}\text { Eighteen-item USDA } \\
\text { HFSSM }\end{array}$ & $\begin{array}{l}\text { Twelve-item CES-D } \\
\text { scale }\end{array}$ & NA & $\begin{array}{l}16.1 \% \text { maternal } \\
\text { depression }\end{array}$ & $\begin{array}{l}\text { Mothers' depression was significantly associated with } \\
\text { subsequent } \mathrm{FI}(\mathrm{aOR} \phi=1.50,95 \% \mathrm{Cl} 1.06,2 \cdot 12) \\
\text { o WIC participation modified relationship }(P=0.005) \text {. } \\
\text { Depressed mothers who received WIC at baseline } \\
\text { were significantly more likely to report } \mathrm{FI} \text { at follow-up } \\
\text { (aOR }=1.59,95 \% \mathrm{Cl} 1.15,2.21) \text {. Depressed mothers } \\
\text { who did not receive WIC were significantly less likely } \\
\text { to report } \mathrm{Fl} \text { at follow-up (aOR }=0.20,95 \% \mathrm{Cl} 0.04,0.93) \\
\text { o SNAP did not significantly modify the relationship } \\
\text { between maternal depression and } \mathrm{FI}(P=0.55)\end{array}$ \\
\hline \multicolumn{9}{|c|}{ Effect of food insecurity on emotional well-being } \\
\hline $\begin{array}{l}\text { Kim and Frongillo } \\
(2007)^{(20)}\end{array}$ & $\begin{array}{l}\text { T1: } 1995-1996 \\
\text { T2: } 1998 \\
\text { T3: } 2000 \\
\text { T4: } 2002\end{array}$ & $\begin{array}{l}\text { Health and Retirement Study (HRS), } \\
\text { Asset and Health Dynamics } \\
\text { among the Oldest Old (AHEAD) }\end{array}$ & $\begin{array}{l}\text { HRS: } \\
\text { Elders, } n 9481 \\
\% \text { Female }=47.7 \% \\
\text { Mean age }=60.8 \text { (sD } 4.2 \text { ) years } \\
\text { Race/ethnicity }=81.2 \% \text { white, } \\
\quad 18.8 \% \text { non-white } \\
\text { Mean income/household } \\
\text { poverty }=\$ \text { US } 52602 \\
\text { (sD } 74783 \text { ) } \\
\text { AHEAD: } \\
\text { Elders, } n 6354 \\
\% \text { Female }=59.9 \\
\text { Mean age }=79.6 \text { (sD } 5 \cdot 8 \text { ) years } \\
\text { Race/ethnicity }=87.4 \% \text { white, } \\
12.6 \% \text { non-white } \\
\text { Mean income/household } \\
\text { poverty }=\$ \text { US } 27760 \\
\text { (sD } 40088 \text { ) }\end{array}$ & $\begin{array}{l}\text { Two-item USDA } \\
\text { HFSSM Module }\end{array}$ & $\begin{array}{l}\text { Eight-item CES-D } \\
\text { scale }\end{array}$ & $\begin{array}{l}\text { HRS: } 8.4 \% \\
\text { AHEAD: } 6.7 \%\end{array}$ & $\begin{array}{l}\text { HRS: } 26.0 \% \\
\text { depressed } \\
\text { AHEAD: } 21.0 \% \\
\text { depressed }\end{array}$ & 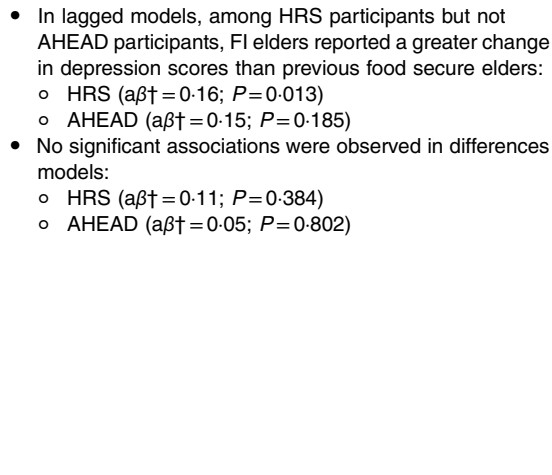 \\
\hline $\begin{array}{l}\text { Zaslow et al. } \\
(2009)^{(19)}\end{array}$ & $\begin{array}{l}\text { T1: 2001-2002 } \\
\text { (9 months) } \\
\text { T2: 2003-2004 } \\
\text { (24 months) }\end{array}$ & $\begin{array}{l}\text { Early Childhood Longitudinal Study- } \\
\text { Birth Cohort }\end{array}$ & $\begin{array}{l}n 8944 \\
\% \text { Female }=48 \cdot 9 \\
\text { Mean age }=\text { child: } 24.4 \text { (SD 2.5) } \\
\quad \text { months }\end{array}$ & $\begin{array}{l}\text { Eighteen-item USDA } \\
\text { HFSSM }\end{array}$ & $\begin{array}{l}\text { Children: Toddler } \\
\text { Attachment Sort } \\
\text { (TAS)-45 } \\
\text { Mothers: Twenty-item } \\
\text { CES-D scale }\end{array}$ & $12.5 \%$ & $\begin{array}{l}\text { Toddler social and } \\
\text { emotional well- } \\
\text { being (TAS): } 38.7 \% \\
\text { Maternal depression: } \\
5.1 \text { (SD 11.7) \% }\end{array}$ & $\begin{array}{l}\text { - FI was significantly related to subsequent maternal } \\
\text { depression }(a \beta \dagger=0.183 ; P<0.001) \text {, which was related } \\
\text { to positive parenting practices }(a \beta \dagger=-0.031 ; P<0.05) \\
\text { o Maternal depression was not significantly related to } \\
\text { toddler emotional well-being }(a \beta \dagger=0.002 ; P>0.05)\end{array}$ \\
\hline
\end{tabular}


Table 2 Continued

\begin{tabular}{|c|c|c|c|c|c|c|c|c|}
\hline \multirow[b]{2}{*}{ Study } & \multirow[b]{2}{*}{ Study time points } & \multirow[b]{2}{*}{ Data set } & \multirow[b]{2}{*}{ Sample and demographics } & \multicolumn{2}{|c|}{ Measures } & \multicolumn{2}{|c|}{ Prevalence at baseline } & \multirow[b]{2}{*}{ Outcomes } \\
\hline & & & & $\mathrm{FI}$ & Emotional well-being & $\mathrm{Fl}$ & Emotional well-being & \\
\hline & & & $\begin{array}{l}\text { Race/ethnicity }=\text { child: } 43.1 \% \text { white, } \\
15.9 \% \text { black, } 20.2 \% \text { Hispanic, } \\
20.9 \% \text { other } \\
\text { Mean income/household } \\
\text { poverty }=25.8 \% \text { had }<1: 00 \\
\text { poverty ratio }\end{array}$ & & & & & $\begin{array}{l}\text { - Positive parenting practice was related to toddler } \\
\text { emotional well-being }(a \beta \dagger=-0.045 ; P<0.05)\end{array}$ \\
\hline $\begin{array}{l}\text { Huang et al. } \\
(2010)^{(22)}\end{array}$ & $\begin{array}{l}1997 \text { (children } \\
\text { ages 3-12 } \\
\text { years) and } \\
2002\end{array}$ & $\begin{array}{l}\text { Child Development Supplement to } \\
\text { the Panel Study of Income } \\
\text { Dynamics }\end{array}$ & $\begin{array}{l}n 416 \\
\text { Child: \% female }=54.69 \\
\text { Child: mean age }=7.46 \text { (sD 2.87) } \\
\text { years } \\
\text { Child: race/ethnicity: } 29.89 \% \text { AA } \\
\text { HoH: \% female }=56.76 \\
\text { HoH: mean age }=36.91 \text { (sD } 7.05 \text { ) } \\
\text { years } \\
\text { Mean income/household } \\
\text { poverty }=\$ \text { US } 24480 \\
\text { (sD } 14670 \text { ) }\end{array}$ & $\begin{array}{l}\text { Eighteen-item USDA } \\
\text { HFSSM }\end{array}$ & $\begin{array}{l}\text { Child: Thirty-two-item } \\
\text { Behavior Problem } \\
\text { Index } \\
\text { Parent stress: Seven- } \\
\text { item index on } \\
\text { caregivers' } \\
\text { perceptions on } \\
\text { feelings and } \\
\text { perceptions of } \\
\text { caring for the child } \\
\text { Parenting distress: } \\
\text { Six-item } \\
\text { psychological } \\
\text { distress scale }\end{array}$ & 1.66 (sD 2.48) & $\begin{array}{l}\text { Child internalizing } \\
\text { behaviours: } 2 \cdot 40 \\
\text { (sD 2.43) } \\
\text { Child externalizing } \\
\text { behaviours: } 5.22 \\
\text { (sD 3.52) } \\
\text { Parental stress: } 1.95 \\
\text { (sD 0.64) } \\
\text { Parental distress: } 4.33 \\
\text { (SD 4.05) }\end{array}$ & $\begin{array}{l}\text { - In lagged models, } \mathrm{FI} \text { was not independently related to } \\
\text { subsequent child internalizing }(\beta=0.17 ; P>0.10) \text { or } \\
\text { externalizing behaviours }(\beta=0.22 ; P<0.10)\end{array}$ \\
\hline $\begin{array}{l}\text { Zilanawala and } \\
\text { Pilkauskas } \\
(2012)^{(15)}\end{array}$ & $\begin{array}{l}\text { T1: } 2001-2003 \\
(3 \text { years }) \\
\text { T2: 2003-2005 } \\
(5 \text { years })\end{array}$ & $\begin{array}{l}\text { Fragile Families and Child } \\
\text { Well-Being Study }\end{array}$ & $\begin{array}{l}n 2825 \\
\% \text { Female }=100 \\
\text { Mothers' mean age }=25.51 \text { years } \\
\text { Race/ethnicity }=16.9 \% \text { white, } \\
50.1 \% \text { black, } 29.9 \% \text { Hispanic } \\
\text { Mean income/household } \\
\text { poverty }=\text { income:needs ratio } \\
1.69 \text { (sD } 1.54 \text { ) }\end{array}$ & $\begin{array}{l}\text { In the past } 12 \text { months, } \\
\text { did you receive free } \\
\text { food or meals? } \\
\text { Subsequent analysis } \\
\text { utilized eighteen- } \\
\text { item USDA HFSSM }\end{array}$ & $\begin{array}{l}\text { Child Behavior and } \\
\text { Adaptive Social } \\
\text { Behavior Inventory }\end{array}$ & $12 \%$ & $\begin{array}{l}17.5 \% \text { (depression) } \\
6.5 \% \text { (anxiety) }\end{array}$ & 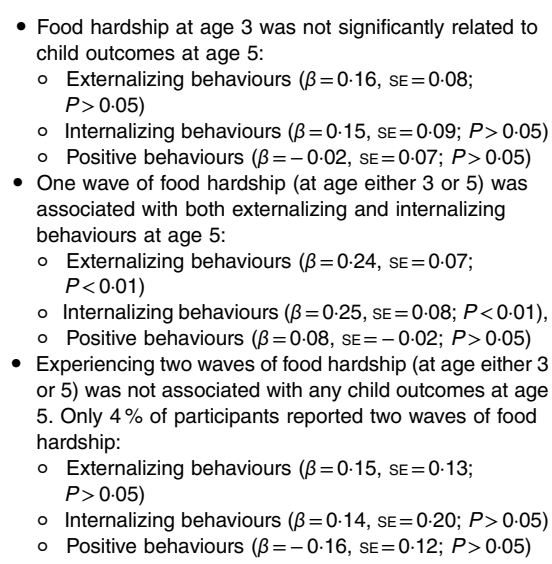 \\
\hline $\begin{array}{l}\text { Laraia et al. } \\
(2015)^{(21)}\end{array}$ & $\begin{array}{l}\text { T1: } 24-29 \text { weeks' } \\
\text { gestation } \\
\text { T2: } 3 \text { months } \\
\text { postpartum } \\
\text { T3: } 12 \text { months } \\
\text { postpartum } \\
\text { during } \\
\text { 2000-2006 }\end{array}$ & $\begin{array}{l}\text { Pregnancy, Infection, and Nutrition } \\
\text { Study }\end{array}$ & $\begin{array}{l}n 526 \\
\% \text { Female }=100 \\
\text { Mean age }{ }^{*}=30.05 \text { years } \\
\text { Race/ethnicity* }=88.0 \% \text { other and } \\
\text { white, } 12.0 \% \text { black } \\
\text { Mean income } / \text { household } \\
\text { poverty }{ }^{*}=442.5 \text { poverty index }\end{array}$ & $\begin{array}{l}\text { Eighteen-item USDA } \\
\text { HFSSM at baseline } \\
\text { Six-item USDA HFS } \\
\text { screener at } \\
\text { 12-months } \\
\text { postpartum }\end{array}$ & $\begin{array}{l}\text { Ten-item Perceived } \\
\text { Stress Scale (PSS) }\end{array}$ & $4.7 \%$ & NA & 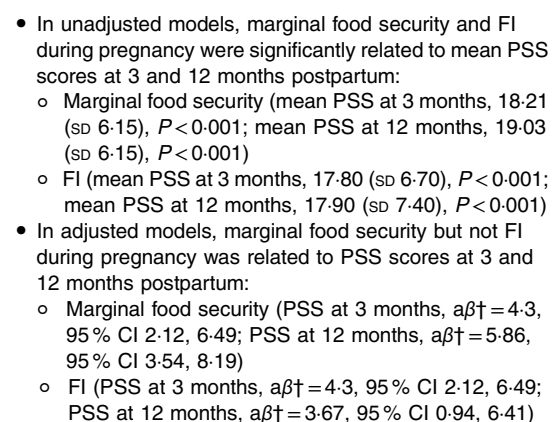 \\
\hline
\end{tabular}


Table 2 Continued

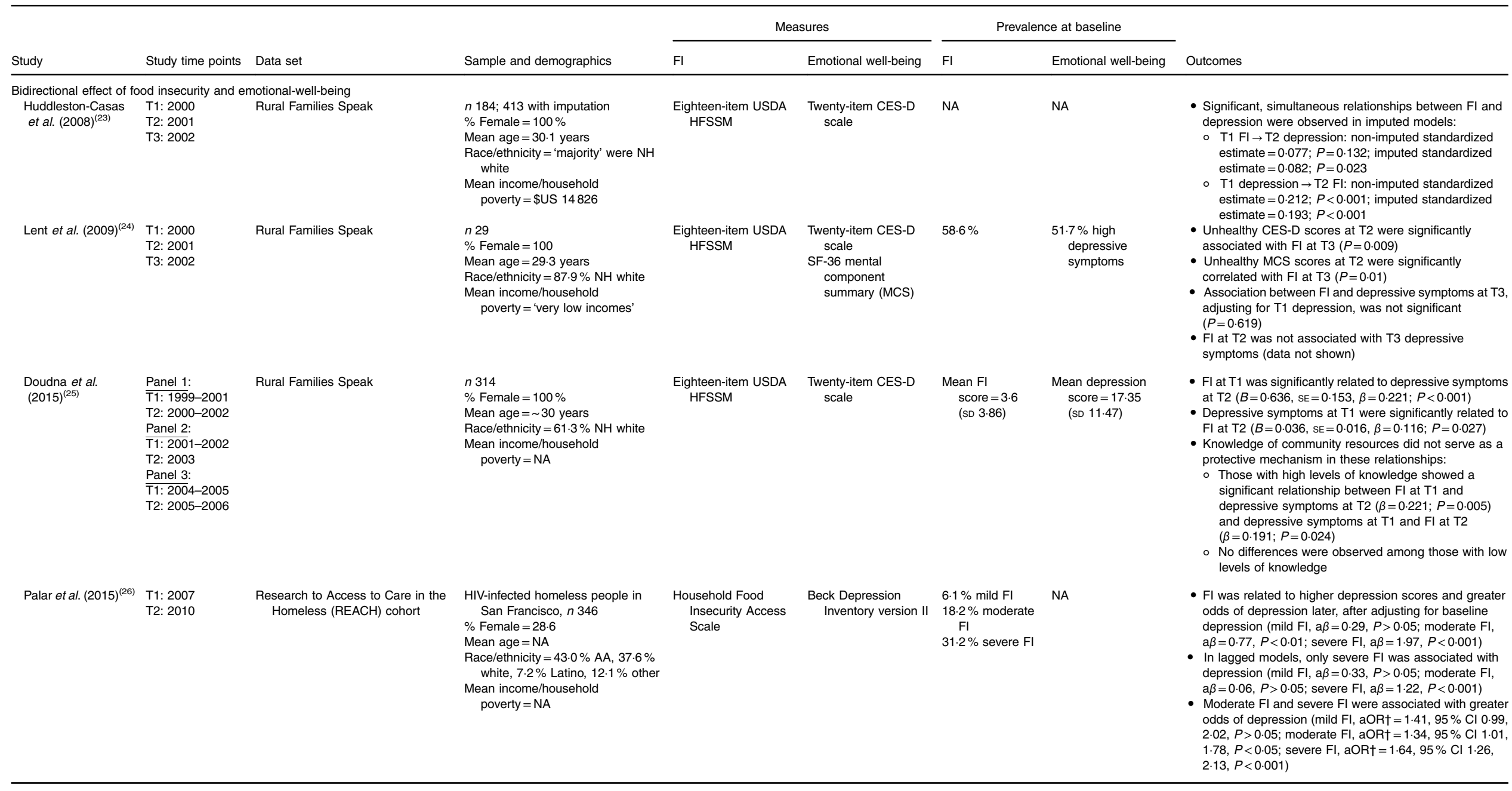

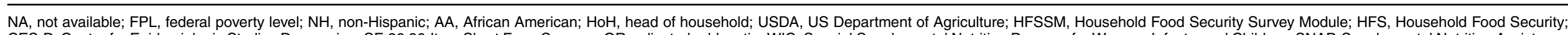

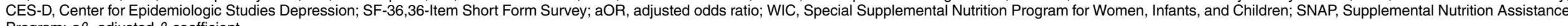
Program; $a \beta$, adjusted $\beta$ coefficient.

†Adjusted estimate: indicates if models controlled for sociodemographics and/or other confounders. 
were food insecure were more likely to show signs of depression compared with those who were food secure $(\beta=0.16 ; P<0.013)$; however, among a population of older elders (mean age $=79.6$ years), the relationship was not significant $(\beta=0.15 ; P=0 \cdot 185)^{(20)}$. Household food insecurity was also associated with subsequent maternal depression (adjusted $\beta=0.183 ; P<0.001$ ) and was mediated by positive parenting practices ${ }^{(19)}$.

In the remaining studies, the association between food insecurity and subsequent emotional well-being was null or was lost after adjusting for additional variables ${ }^{(15,22)}$. For instance, Zilanawala and Pilkauskas found in their crosssectional analysis that food insecurity was positively associated with children's externalizing and internalizing behaviours, but longitudinally this association did not persist $^{(15)}$. Huang et al. also reported that food insecurity was associated with children's internalizing and externalizing behaviours, but after adjusting for parental characteristics, the relationship was no longer statistically significant $^{(22)}$

\section{Bidirectional effect of food insecurity and emotional well-being}

Four articles examined the bidirectional relationship between emotional well-being and food insecurity ${ }^{(23-26)}$. Three of these studies examined maternal populations $^{(23-25)}$, and the last examined HIV-infected homeless people in San Francisco ${ }^{(26)}$. All four studies examined depression using the CES-D ${ }^{(23-25)}$ or the Beck Depression Inventory ${ }^{(26)}$. One study also examined overall mental health ${ }^{(24)}$; the three studies that used the CES-D assessed maternal depression ${ }^{(23-25)}$. Food insecurity was determined with the USDA HFSSM in three studies ${ }^{(23-25)}$, while one study measured food insecurity with the Household Food Insecurity Access Scale ${ }^{(26)}$. The sample size in these studies was in the range of 29-346 with a mean sample size of 218.

Huddleston-Casas et al. examined non-imputed and imputed relationships. In the non-imputed findings, time 1 food insecurity was not significantly related to time 2 depression $(\beta=0.077 ; P=0 \cdot 132)$, but time 1 depression was significantly related to time 2 food insecurity $(\beta=0 \cdot 212$; $P<0.001)^{(23)}$. In imputed models, both the relationship between food insecurity and subsequent depression $(\beta=0.082 ; \quad P=0.023)$ and the relationship between depression and subsequent food insecurity were found to be statistically significant $(\beta=0 \cdot 193 ; P<0 \cdot 001)^{(23)}$.

Lent et al. reported that lower mental health scores at time 2 were significantly related to food insecurity at time $3(P=0.01)^{(24)}$. Post boc $\chi^{2}$ analyses showed a nonsignificant relationship between food insecurity and continuation of depressive symptoms at time 3 among the participants who scored at risk of depression at time 1 $(P=0.62)^{(24)}$. Doudna et al. also examined food insecurity and emotional well-being across two time periods ${ }^{(25)}$. The results from their study indicated statistically significant associations between food insecurity at time 1 predicting time 2 depressive symptoms $(\beta=0.221 ; P<0.001)$, and depressive symptoms at time 1 predicting time 2 food insecurity $(\beta=0.116 ; P=0.027)^{(25)}$. These authors also examined how community resources may have mediated the bidirectional models, and found that knowledge of community resources was not protective in either relationship ${ }^{(25)}$. Finally, Palar et al. examined the bidirectional relationship between the severity of food insecurity (mild $v$. moderate $v$. severe food insecurity) and depression ${ }^{(26)}$. Moderate and severe food insecurity were significantly related to higher depression scores and greater odds of depression later, after adjusting for time 1 depression $(P<0.01)^{(26)}$. In lagged models to control for the potential of reverse causality, only severe food insecurity was associated with depression $(P<0 \cdot 001)$, suggesting possible reverse causality for mild and moderate food insecurity and depression severity ${ }^{(26)}$.

\section{Discussion}

The majority of studies included in the present review show significant associations between poor mental health and food insecurity, and suggest a bidirectional association whereby food insecurity increases the risk of poor emotional health, and poor emotional health increases the risk of food insecurity. Two-thirds (66.7\%) of the nine studies assessing food insecurity at baseline found positive associations with poor emotional health at followup $^{(19-21,23,25,26)}$. Likewise, $100 \%$ of the seven studies measuring poor emotional health at baseline showed positive associations with food insecurity at follow-up ${ }^{(16-18,23-26)}$.

These findings, while perhaps not surprising, are indeed alarming. Poor emotional health is an adverse outcome within itself and greatly impacts quality of life. Independently, poor emotional health can lead to poor physical health outcomes, including CVD, type 2 diabetes, HIV/ AIDS, tuberculosis, physical disability, unintentional and intentional injury, impaired child growth and development, and infant mortality ${ }^{(27)}$. Similarly, food insecurity has been associated with asthma, anaemia, birth defects, diabetes, hypertension, hyperlipidaemia, poor sleep outcomes and oral health problems ${ }^{(28)}$. When both poor emotional health and food insecurity are experienced by the same individual, these effects on physical health may be interactive and multiplicative. It is therefore necessary to identify those individuals, families and communities that are at risk for poor emotional health, food insecurity, or both. For example, federally funded food programmes such as the Supplemental Nutrition Assistance Program (SNAP) and the Special Supplemental Nutrition Program for Women, Infants, and Children (WIC) - whose purpose is to help lift Americans out of food insecurity - can also promote mental and emotional well-being through social marketing campaigns and messaging, screenings and referrals. In fact, some WIC programmes in California and Washington, DC 
have already successfully integrated postpartum depression screening and referrals into their nutrition intake and assessment protocol ${ }^{(29,30)}$. The prevention of poverty and other efforts to address materially impoverished conditions among at-risk groups would likely improve both food insecurity and emotional well-being.

Conversely, physicians, mental health professionals, social workers, registered dietitians and hospitals should include individual and household food security screenings as part of their intake and admissions protocol. Hager et al. have validated a two-item food security screening tool that can serve as a quick and easy screen for food insecurity risk among low-income families with young children, which has subsequently been used and validated in adults and youth ${ }^{(31,32)}$. It is important that positive screening is followed with an appropriate intervention. For patients with a positive screen, health-care professionals can then make appropriate and timely referrals to federally funded food programmes that patients may be eligible for, including SNAP, WIC, school meals programmes, and local pantries and soup kitchens. Additionally, hospitals can conduct on-site SNAP enrolment, serve as a WIC site, operate food pantries and summer meals programmes, and offer access to fresh fruits and vegetables via farmers' markets and hospital gardens ${ }^{(33)}$. Intervention studies are needed to assess if programmes addressing food insecurity improve mental health and vice versa. Research on the long-term additive effect of food insecurity and poor emotional well-being on quality of life and interpersonal relationships is also needed. To support this study and the study among low-income countries ${ }^{(14)}$, similar studies are needed across more middle-income countries.

Despite the consistent patterns found among most of the studies reviewed, a few limitations that should be noted. First, several studies included small or homogeneous samples, limiting generalizability within studies. However, across the twelve studies, sample sizes ranged from 29 to 9481, and samples varied by age and geographic location within the USA. It is important to note that many of the studies included in the present review were secondary data analyses, and therefore were not designed to rigorously examine the relationships between food insecurity and emotional well-being. Most of these studies examined maternal populations. As such, we need a better understanding of how food insecurity and emotional health impact other populations such as children and older adults. Often, in those studies where the relationship between food insecurity and emotional well-being was of primary interest, the studies were small and may have been underpowered. Despite this, the results were consistent across populations and across study design limitations. Additionally, we limited our search to the past 10 years to gain an understanding of the relationships post-recession; there may be additional relevant studies conducted prior to this period. Another limitation lies in the large number of measurement tools and outcome measures analysed by the studies, which unfortunately does not allow for metaanalysis. For example, while food insecurity was most commonly measured by the USDA HFSSM, some studies determined food hardship or hunger using different instruments. Similarly, emotional health outcomes included depression, anxiety and perceived stress, among others, and each of these outcomes was measured with several different scales. Such diversity in outcomes and measures makes it difficult to compare findings across studies. Still, trends in the longitudinal associations between poor emotional health and food insecurity (and vice versa) appear consistent regardless of these differences.

Notably, all twelve studies focused on the association between food insecurity and negative emotional health outcomes (depression, stress, anxiety, etc.). Despite our range of emotional health search terms, not one study meeting our inclusion and exclusion criteria measured positive emotional health outcomes, such as happiness, satisfaction or contentment. While it may be assumed from the current review that these emotions would be inversely associated with food insecurity, there is a need for research to study these relationships. Focusing solely on negative emotions overlooks the potential for building on strengths and resiliency of the populations being studied. Conversely, other phenomena that may impact the health and well-being of populations struggling with food insecurity, such as racism, discrimination, abuse, stigma and social support, are worth exploring.

\section{Conclusions}

The present systematic narrative review identified a bidirectional relationship between poor mental health and food insecurity. Public health practitioners addressing mental health may consider screening for food insecurity for at-risk populations and vice versa. Better-constructed studies are needed to follow cohorts at risk for both food insecurity and poor emotional health to further understand the mediators and moderators of the relationships. Intervention studies designed to mitigate or reverse risks are also needed to determine best evidence for practice and policy.

\section{Acknowledgements}

Financial support: This research received no specific grant from any funding agency in the public, commercial or not-for-profit sectors. Conflict of interest: There are no conflicts of interest to disclose. Authorship: All authors contributed substantially to the manuscript. J.B.R.C. compiled the titles. All authors reviewed titles, abstracts and full-text articles. All authors were involved in the analysis and writing of the manuscript. Ethics of buman subject participation: As a systematic review, no new human subjects' data were collected. 


\section{Supplementary material}

To view supplementary material for this article, please visit https://doi.org/10.1017/S1368980017002221

\section{References}

1. Coleman-Jensen A, Rabbitt M, Gregory C et al. (2016) Household Food Insecurity in the United States in 2015. Economic Research Report no. ERR-215. https://www.ers. usda.gov/webdocs/publications/79761/err-215.pdf?v=42636 (accessed September 2016).

2. US Department of Agriculture, Economic Research Service (2015) Key statistics \& graphics. http://www.ers.usda.gov/ topics/food-nutrition-assistance/food-security-in-the-us/keystatistics-graphics/ (accessed September 2016).

3. Roshanafshar S \& Hawkins E (2015) Health at Glance Food Insecurity in Canada. Catalogue no. 82-624-X. http:// www5.statcan.gc.ca/olc-cel/olc.action?lang $=$ en\&ObjId=82-624X201500114138\&ObjType $=47$ (accessed September 2016).

4. Center on Hunger and Poverty (2003) The Paradox of Hunger and Obesity in America. Waltham, MA: Brandeis University.

5. Jyoti DF, Frongillo EA \& Jones SJ (2005) Food insecurity affects school children's academic performance, weight gain, and social skills. J Nutr 135, 2831-2839.

6. Bhattacharya J, Currie J \& Haider S (2004) Poverty, food insecurity, and nutritional outcomes in children and adults. $J$ Health Econ 23, 839-862.

7. Chilton M, Black MM, Berkowitz C et al. (2009) Food insecurity and risk of poor health among US-born children of immigrants. Am J Public Health 99, 556-562.

8. Seligman HK, Bindman AB, Vittinghoff E et al. (2007) Food insecurity is associated with diabetes mellitus: results from the National Health Examination and Nutrition Examination Survey (NHANES) 1999-2002. J Gen Intern Med 22, 1018-1023.

9. Berkowitz SA, Baggett TP, Wexler DJ et al. (2013) Food insecurity and metabolic control among US adults with diabetes. Diabetes Care 36, 3093-3099.

10. Hromi-Fiedler A, Bermúdez-Millán A, Segura-Pérez S et al. (2011) Household food insecurity is associated with depressive symptoms among low-income pregnant Latinas. Matern Child Nutr 7, 421-430.

11. Sharkey JR, Johnson CM \& Dean WR (2011) Relationship of household food insecurity to health-related quality of life in a large sample of rural and urban women. Women Health 51, 442-460.

12. Feeny S, McDonald L \& Posso A (2014) Are poor people less happy? Findings from Melanesia. World Dev 64, 448-459.

13. Alaimo K, Olson CM \& Frongillo EA (2002) Family food insufficiency, but not low family income, is positively associated with dysthymia and suicide symptoms in adolescents. J Nutr 132, 719-725.

14. Weaver LJ \& Hadley C (2009) Moving beyond hunger and nutrition: a systematic review of the evidence linking food insecurity and mental health in developing countries. Ecol Food Nutr 48, 263-284.

15. Zilanawala A \& Pilkauskas NV (2012) Material hardship and child socioemotional behaviors: differences by types of hardship, timing, and duration. Child Youth Serv Rev 34, 814-825.

16. Hanson KL \& Olson CM (2012) Chronic health conditions and depressive symptoms strongly predict persistent food insecurity among rural low-income families. $J$ Health Care Poor Underserved 23, 1174-1188.

17. Garg A, Toy S, Tripodis Y et al. (2015) Influence of maternal depression on household food insecurity for low-income families. Acad Pediatr 15, 305-310.

18. Hernandez DC, Marshall A \& Mineo C (2013) Maternal depression mediates the association between intimate partner violence and food insecurity. $J$ Womens Health $\mathbf{2 3}$, 29-37.

19. Zaslow M, Bronte-Tinkew J, Capps R et al. (2009) Food security during infancy: implications for attachment and mental proficiency in toddlerhood. Matern Child Health J 13, 66-80.

20. Kim K \& Frongillo EA (2007) Participation in food assistance programs modifies the relation of food insecurity with weight and depression in elders. J Nutr 137, 1005-1010.

21. Laraia B, Vinikoor-Imler LC \& Siega-Riz AM (2015) Food insecurity during pregnancy leads to stress, disordered eating, and greater postpartum weight among overweight women. Obesity (Silver Spring) 23, 1303-1311.

22. Huang J, Matta Oshima KM \& Kim Y (2010) Does food insecurity affect parental characteristics and child behavior? Testing mediation effects. Soc Serv Rev 84, 381-401.

23. Huddleston-Casas C, Charnigo R \& Simmons LA (2008) Food insecurity and maternal depression in rural, lowincome families: a longitudinal investigation. Public Health Nutr 12, 1133-1140.

24. Lent MD, Petrovic LE, Swanson JA et al. (2009) Maternal mental health and the persistence of food insecurity in poor rural families. J Health Care Poor Underserved 20, 645-661.

25. Doudna KD, Reina AS \& Greder KA (2015) Longitudinal associations among food insecurity, depressive symptoms, and parenting. J Rural Mental Health 39, 178-187.

26. Palar K, Kushel M, Frongillo EA et al. (2015) Food insecurity is longitudinally associated with depressive symptoms among homeless and marginally-housed individuals living with HIV. AIDS Behav 19, 1527-1534.

27. Prince M, Patel V, Saxena S et al. (2007) No health without mental health. Lancet 370, 859-877.

28. Gundersen C \& Ziliak JP (2015) Food insecurity and health outcomes. Health Aff (Millwood) 34, 1830-1839.

29. Pooler J, Perry DF \& Ghandour RM (2013) Prevalence and risk factors for postpartum depressive symptoms among women enrolled in WIC. Matern Child Health $J$ 17, 1969-1980.

30. Fritz BJ (2015) Screening for perinatal depression at county WIC offices. J Obstet Gynecol Neonatal Nurs 44, S13.

31. Baer TE, Scherer EA, Fleegler EW et al. (2015) Food insecurity and the burden of health-related social problems in an urban youth population. $J$ Adolesc Health 57, 601-607.

32. Gundersen C, Engelhard EE, Crumbaugh AS et al. (2017) Brief assessment of food insecurity accurately identifies high-risk US adults. Public Health Nutr 20, 1367-1371.

33. Share Our Strength (2012) Fighting hunger through healthcare: a seamless solution. https://bestpractices. nokidhungry.org/content/fighting-hunger-through-healthcare (accessed September 2016). 